\title{
DFT performance in the IQA energy partition of small water clusters
}

Fernando Jiménez-Grávalos ${ }^{\mathrm{a}}$, José Luis Casals-Sainz ${ }^{\mathrm{a}}$, Evelio Francisco ${ }^{\mathrm{a}}$, Tomás Rocha-Rinza ${ }^{\mathrm{b}}$, Ángel Martín Pendás ${ }^{\mathrm{a}}$, José Manuel Guevara-Vela ${ }^{\mathrm{a}, *}$

${ }^{a}$ Departament of Analytical and Physical Chemistry, University of Oviedo, E-33006, Oviedo, Spain.

${ }^{b}$ Institute of Chemistry, National Autonomous University of Mexico, Circuito Exterior, Ciudad Universitaria, Delegación Coyoacán C.P. 04510, Mexico City, Mexico.

\begin{abstract}
This paper addresses an assessment of the performance of a large set of exchange-correlation functionals in the description of hydrogen bonding within the Interacting Quantum Atoms (IQA) energy partition. Specifically, we performed IQA analyses over a series of small water clusters $\left(\mathrm{H}_{2} \mathrm{O}\right)_{\mathrm{n}}$ with $\mathrm{n} \leq 6$. Apart from LDA-like approximations, all the considered families of exchange-correlation functionals (GGA, meta-GGA, and hybrid) reproduce the trends associated with hydrogen-bond non-additive effects computed with reference Møller-Plesset and coupled cluster wave functions. In other words, the IQA energy partition together with most of the functionals addressed herein produce good results concerning the study of non-additivity in hydrogen bonds at a reduced cost as compared with correlated wave functions approximations. This conditions might be further exploited in the examination of larger hydrogen-bonded complexes.
\end{abstract}

Keywords:

Quantum theory of atoms in molecules, Interacting quantum atoms, Density functional theory

\section{Introduction}

Density Functional Theory (DFT) is regarded as one of the most fruitful theories in computational chemistry. [1] Its widespread use arises from its convenient compromise between accuracy and computational cost. [2] DFT has been applied to a wide range of problems, from the study of small chemical systems to the analysis of large macromolecular aggregates. [3] Indeed, the fields of application of DFT increases continuously: it is utilised in the understanding of catalytic processes, the discovery of new drugs, and the development of new materials. $[4,5]$

${ }^{*}$ To whom correspondence should be addressed: jmguevarav@gmail.com 
Besides these applications, DFT also provides the basis for the understanding and exploitation of concepts such as electronegativity and chemical hardness which are very useful in many branches of chemistry. [6] The development of these concepts in the field of "conceptual DFT" has provied a series of tools for the interpretation of molecular properties, in particular, those concerned with chemical reactivity. $[7,8]$

Likewise to conceptual DFT, the topological analysis of the electron density in accordance with the Quantum Theory of Atoms in Molecules (QTAIM) has resulted in new insights about molecules and molecular clusters as well as the processes undergone by these systems. $[9,10]$ The charge distribution is not the only scalar field that can be examined in this way. Many other functions, e.g. the electron localisation function, the source function or the virial field have been studied with similar topological approaches than that employed in the QTAIM. [1113] The investigation concerning the properties of these scalar fields and its applications has resulted in the emergence of the field of theoretical chemistry known as Quantum Chemical Topology (QCT). [14]

The Interacting Quantum Atoms (IQA) $[15,16]$ energy partition is a notable method for wave function analyses. This approach allows for the separation of the total electronic energy of a system into physically-meaningful intra- and inter-atomic contributions. Initially, IQA could only be applied to small systems due to the high computational burden required to perform this type of partition of the electronic energy. Moreover, the set of available electronic structure methods which could be coupled to IQA was limited to HF, CASSCF, CISD, FCI or CCSD. Popelier and coworkers have however recently used IQA with the B3LYP functional, [17] and some of us have extended this energy partition to the rest of the most common exchange-correlation functionals. [18] The coupling of IQA and DFT reduces dramatically the computational cost when an effective treatment of electron correlation is needed and, therefore, it opens new possibilities for the applicability of the IQA analysis.

One of such opportunities is the study of the non-additivity in hydrogen bonding in water clusters. These adducts are prototypical systems for the rationalisation of cooperative and anticooperative effects in hydrogen bonding. For example, we have used IQA coupled with Hartree-Fock and correlated wavefunctions to study cooperativity and anticooperativity in $\left(\mathrm{H}_{2} \mathrm{O}\right)_{\mathrm{n}}[19,20]$ clusters with $\mathrm{n} \leq 6$. These investigations lead to a hydrogen bond strength hierarchy within water clusters in terms of the single and double character of the hydrogen bonding donor and acceptor $\mathrm{H}_{2} \mathrm{O}$ monomers. We present herein an assessment of the perfor- 
mance of sixteen different functionals in the description of the non-additivity of hydrogen bond in the small water clusters shown in Figure (1) with the IQA energy partition. Hereof, we note that DFT describes hydrogen-bonded systems appropriately. On this subject, Michaelides et al. [21] concluded that the general DFT description of water is acceptable and that hybrid functionals performed the best in accounting for monomer properties and the non-additivity of hydrogen bonding. However, Mendedev et al. showed that for many new hybrid functionals the corresponding densities deviate from the exact ones because they sacrifice physical rigour for the flexibility of empirical fitting. [22]
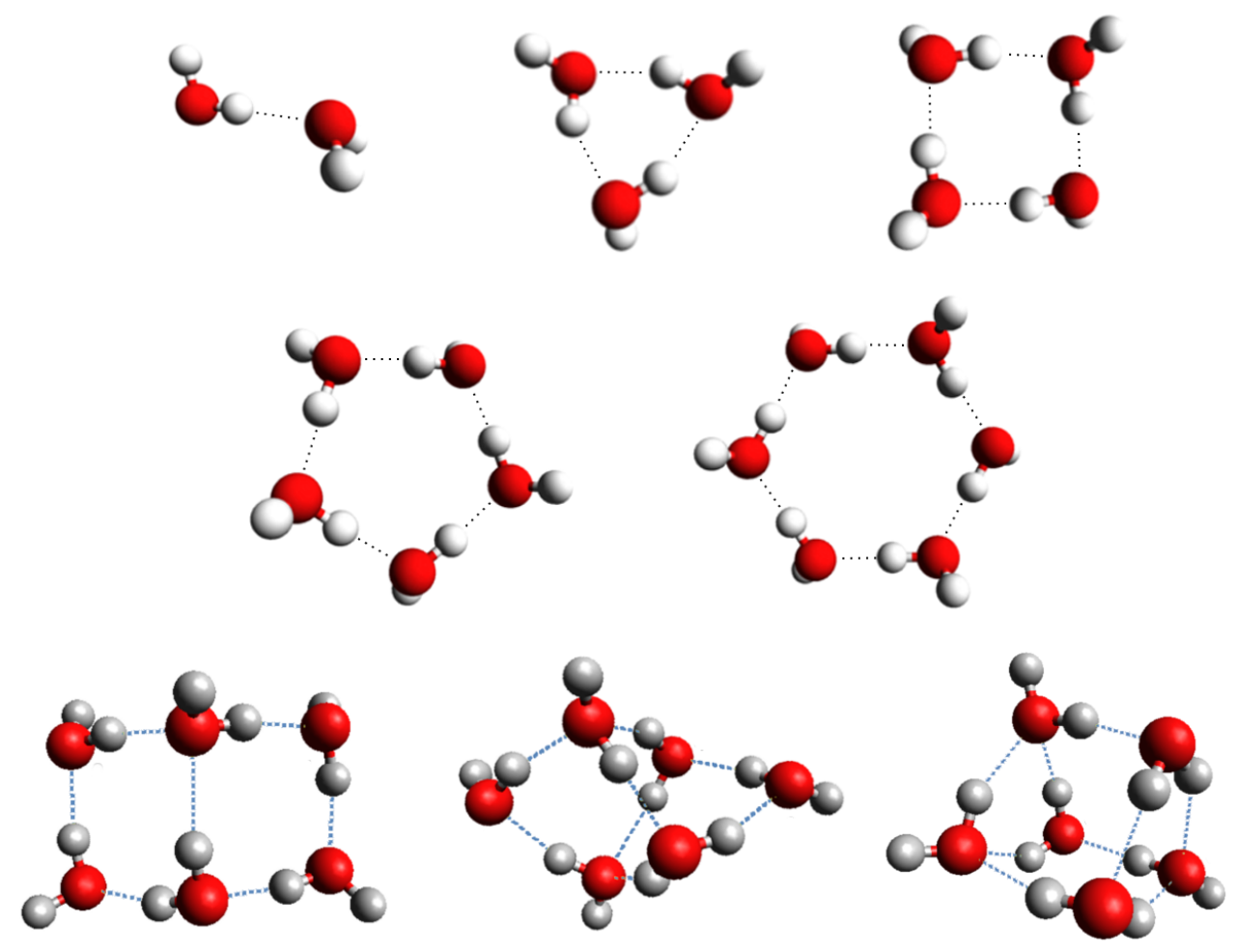

Figure 1: Small water clusters used as models in the present research.

Given this context, the present manuscript is structured as follows. First, we present a brief outline of the IQA partition and an strategy for its application to DFT electronic energies, emphasising the division of the exchange-correlation contribution. Later, we address the computational details employed in this investigation to finally discuss the main results of this work. Overall, we found that IQA/DFT analyses based on most of the functionals considered herein describes suitably the non-additive effects previously described, thus paving the way for future studies on relatively large hydrogen bonded systems. 


\section{Interacting Quantum Atoms approach}

The Quantum Theory of Atoms in Molecules (QTAIM) formulated by Bader and his coworkers defines a partition of real space from the electron density of the molecular system under study. [23] This division allows in turn to decompose the total electronic energy $(E)$ into intra and inter-atomic components by using the first (1-RDM) and second order (2-RDM) reduced density matrices. Such an energy decomposition constitutes the Interacting Quantum Atoms (IQA) approach: $[15,16]$

$$
E=\sum_{\mathrm{A}} E_{\mathrm{net}}^{\mathrm{A}}+\sum_{\mathrm{A}} \sum_{\mathrm{A}>\mathrm{B}} E_{\mathrm{int}}^{\mathrm{AB}}
$$

where $E_{\text {net }}^{\mathrm{A}}$ denotes the intra-atomic energy of region $\mathrm{A}$ and $E_{\mathrm{int}}^{\mathrm{AB}}$ corresponds to the interaction energy between domains $\mathrm{A}$ and B. $E_{\text {net }}^{\mathrm{A}}$ is obtained as

$$
E_{\mathrm{net}}^{\mathrm{A}}=T^{\mathrm{A}}+V_{\mathrm{ne}}^{\mathrm{AA}}+V_{\mathrm{ee}}^{\mathrm{AA}}
$$

with $T^{\mathrm{A}}, V_{\mathrm{ne}}^{\mathrm{AA}}$ and $V_{\mathrm{ee}}^{\mathrm{AA}}$ being the kinetic energy of atom A, the nucleus-electron interaction and the electron-electron repulsion within atom A, respectively. Similarly, $E_{\text {int }}^{\mathrm{AB}}$ stands for the sum of the nucleus-nucleus $\left(V_{\mathrm{nn}}^{\mathrm{AB}}\right)$ and the electron-electron $\left(V_{\mathrm{ee}}^{\mathrm{AB}}\right)$ repulsions, together with the attraction between the electrons located in atom $\mathrm{A}$ and the nucleus in atom $\mathrm{B}\left(V_{\mathrm{ne}}^{\mathrm{BA}}\right)$ and vice $\operatorname{versa}\left(V_{\mathrm{ne}}^{\mathrm{AB}}\right)$,

$$
E_{\mathrm{int}}^{\mathrm{AB}}=V_{\mathrm{nn}}^{\mathrm{AB}}+V_{\mathrm{ee}}^{\mathrm{AB}}+V_{\mathrm{ne}}^{\mathrm{AB}}+V_{\mathrm{ne}}^{\mathrm{BA}} .
$$

One attractive feature of IQA is the fact that it is based on orbital-invariant scalar functions which enables the separation of $E$ in terms corresponding to atoms and to atomic pairs in an unambiguous and transparent manner. Another important feature of the IQA methodology in the study of intermolecular interactions is the ability to put together atoms to form groups. These groups, or superbasins, can be identified with functional groups within a molecule, or with molecules within a molecular cluster. We can define in this way the energy of a collection of atoms $\mathscr{G}$ as

$$
E_{\text {net }}^{\mathscr{G}}=\sum_{\mathrm{A} \in \mathscr{G}} E_{\mathrm{net}}^{\mathrm{A}}+\frac{1}{2} \sum_{\mathrm{A} \in \mathscr{G}} \sum_{\substack{B \in \mathscr{G} \\ \mathrm{A} \neq \mathrm{B}}} E_{\mathrm{int}}^{\mathrm{AB}},
$$

while the interaction energy between groups $\mathscr{G}$ and $\mathscr{H}$ reads, 


$$
E_{\text {int }}^{\mathscr{G}, \mathscr{H}}=\sum_{\mathrm{A} \in \mathscr{G}} \sum_{B \in \mathscr{H}} E_{\text {int }}^{\mathrm{AB}} .
$$

The change in energy related with the formation of a molecular cluster from molecules $\mathscr{G} \ldots \mathscr{H} \ldots \mathscr{I}$ can be computed using the formula:

$$
\Delta E=\sum_{\mathscr{G}} E_{\mathrm{def}}^{\mathscr{G}}+\sum_{\mathscr{G}} \sum_{\mathscr{G}>\mathscr{H}} E_{\mathrm{int}}^{\mathscr{G} \mathscr{H}}
$$

wherein $E_{\text {def }}^{\mathscr{G}}$ is the difference of the energy of group $\mathcal{G}$ within the molecular cluster $\mathcal{G} \cdots \mathcal{H} \cdots \mathcal{I}$ and in its isolated state. $[15,20]$ It is possible to modify equation (6) by adding fractions of $E_{\text {def }}^{\mathscr{G}}$ and $E_{\text {def }}^{\mathscr{H}}$ to $E_{\text {int }}^{\mathscr{G}, \mathscr{H}}$. The resulting expression is a pairwise sum $\mathscr{G} \ldots \mathscr{H}$ of interacting monomers.

$$
\begin{aligned}
\Delta E & =\sum_{\mathscr{G}} \sum_{\mathscr{G}>\mathscr{H}}\left(E_{\mathrm{int}}^{\mathscr{G} \mathscr{H}}+\left(\frac{E_{\text {int }}^{\mathscr{H}}}{\sum_{\mathscr{I} \neq \mathscr{G}} E_{\text {int }}^{\mathscr{I} \mathscr{G}}}\right) E_{\mathrm{def}}^{\mathscr{G}}+\left(\frac{E_{\text {int }}^{\mathscr{G}}}{\sum_{\mathscr{I} \neq \mathscr{H}} E_{\text {int }}^{\mathscr{I} \mathscr{H}}}\right) E_{\mathrm{def}}^{\mathscr{H}}\right) \\
& =\sum_{\mathscr{G}} \sum_{\mathscr{G}>\mathscr{H}} E_{\mathrm{int}}^{\mathscr{G} \mathscr{H} !} .
\end{aligned}
$$

The electron-electron term can be further divided into Coulombic and exchange-correlation terms. This division enables a rearrangement of the whole interatomic energy as the addition of classical $\left(V_{\mathrm{cl}}^{\mathrm{AB}}\right)$ and exchange-correlation $\left(V_{\mathrm{xc}}^{\mathrm{AB}}\right)$ contributions,

$$
E_{\mathrm{int}}^{\mathrm{AB}}=V_{\mathrm{cl}}^{\mathrm{AB}}+V_{\mathrm{xc}}^{\mathrm{AB}}
$$

$V_{\mathrm{xc}}^{\mathrm{AB}}$ and $V_{\mathrm{cl}}^{\mathrm{AB}}$ are identified with covalent and ionic components of the interaction between atoms A and B. [16]

\section{IQA implementation of DFT}

The IQA energy decomposition implies the calculation of (i) the kinetic energy, which depends on the 1-RDM, (ii) the Coulombic or classical (cl) interaction energy that is determined solely by the electron density $\rho(\mathbf{r})$ of the system and (iii) the exchange-correlation energy that entails the computation of the corresponding density, $\rho_{\mathrm{xc}}\left(\mathbf{r}_{1}, \mathbf{r}_{2}\right)$, which is related to the pair density $\rho_{2}\left(\mathbf{r}_{1}, \mathbf{r}_{2}\right)$, by the following relationship:

$$
\rho_{2}\left(\mathbf{r}_{1}, \mathbf{r}_{2}\right)=\rho\left(\mathbf{r}_{1}\right) \rho\left(\mathbf{r}_{2}\right)+\rho_{\mathrm{xc}}\left(\mathbf{r}_{1}, \mathbf{r}_{2}\right)
$$


The dependence of the IQA partition on 1-RDM and 2-RDM impedes the direct use of DFT in IQA. This problem had been previously bypassed by assigning the exchange-correlation energy not accounted by the exchange obtained directly from the Kohn-Sham determinant to intra-atomic exchange-correlation terms. This approach leads to the complete recovery of the electronic energy by the IQA partition, although, it overestimates systematically the exchangecorrelation component of $V_{\mathrm{ee}}^{\mathrm{AA}}$ in detriment of $E_{\mathrm{int}}^{\mathrm{AB}} \cdot[17]$

Some of us proposed an alternative approach that uses scaling arguments, which results in a complete recovery of the total electronic energy and a correction of the intra- and interatomic exchange-correlation energies. [18] We describe now this coupling of IQA and DFT, which is the one followed in this work. For a non-hybrid exchange-correlation functional $\epsilon(\mathbf{r})$, the DFT exchange-correlation energy, $E_{\mathrm{xc}}^{D F T}$, can be calculated as

$$
E_{\mathrm{xc}, \mathrm{loc}}^{D F T}=\int_{\infty} \mathrm{d} \mathbf{r} \rho(\mathbf{r}) \epsilon(\mathbf{r}),
$$

For hybrid functionals Equation (10) takes the form

$$
E_{\mathrm{xc}}^{\mathrm{DFT}}=E_{\mathrm{xc}, \mathrm{loc}}^{\mathrm{DFT}}+a_{0} E_{\mathrm{x}}^{\mathrm{KS}}
$$

$a_{0}$ is the fraction of the Hartree-Fock exchange used by the functional $\epsilon_{\mathrm{xc}}$ and $E_{\mathrm{x}}^{\mathrm{KS}}$ is the exchange energy calculated using the Kohn-Sham molecular orbitals.

We define now the quantity $\lambda_{\mathrm{A}}$ as in reference [18]

$$
\lambda_{\mathrm{A}}=a_{0}+\frac{1}{E_{\mathrm{x}}^{\mathrm{A}, \mathrm{KS}}} \int_{\mathrm{A}} \rho(\mathbf{r}) \epsilon(\mathbf{r}),
$$

in which

$$
E_{\mathrm{xc}}^{\mathrm{A}}=E_{\mathrm{xc}}^{\mathrm{AA}}+\frac{1}{2} \sum_{\mathrm{B} \neq \mathrm{A}} E_{\mathrm{xc}}^{\mathrm{AB}}
$$

with

$$
E_{\mathrm{xc}}^{\mathrm{AA}}=\frac{1}{2} \int_{\Omega \mathrm{A}} \mathrm{d} \mathbf{r}_{1} \int_{\Omega \mathrm{A}} \mathrm{d} \mathbf{r}_{2} r_{12}^{-1} \rho_{\mathrm{xc}}\left(\mathbf{r}_{1}, \mathbf{r}_{2}\right),
$$


and

$$
E_{\mathrm{xc}}^{\mathrm{AB}}=\int_{\Omega \mathrm{A}} \mathrm{d} \mathbf{r}_{1} \int_{\Omega \mathrm{B}} \mathrm{d} \mathbf{r}_{2} r_{12}^{-1} \rho_{\mathrm{xc}}\left(\mathbf{r}_{1}, \mathbf{r}_{2}\right) .
$$

Now, we can define intra- $(\mathrm{A}=\mathrm{B})$ and inter-atomic $(\mathrm{A} \neq \mathrm{B})$ IQA/DFT exchange-correlation energies as

$$
\tilde{E}_{\mathrm{xc}}^{\mathrm{AB}}=\frac{1}{2}\left[\lambda_{\mathrm{A}}+\lambda_{\mathrm{B}}\right] V_{\mathrm{x}}^{\mathrm{AB}, \mathrm{KS}}
$$

from which the total DFT exchange correlation energy (LHS of Eq. (11)) can be completely recovered as

$$
E_{\mathrm{xc}}^{\mathrm{DFT}}=\sum_{\mathrm{A}} \tilde{E}_{\mathrm{xc}}^{\mathrm{AA}}+\sum_{\mathrm{A}>\mathrm{B}} \tilde{E}_{\mathrm{xc}}^{\mathrm{AB}}
$$

\section{Computational Details}

We decided to use previously reported CCSD/aug-cc-pVDZ homodromic water cluster structures, comprising from 2 to 6 water molecules, taken from the work of Segarra-Martí et al. [24]. Given that the structure of the monomer was not reported in this reference, we optimised the corresponding geometry using the same level of theory. Additionally, we optimised the structures of other four water hexamers using the same approximation (see Figure 1). From these geometries, we procured the electron density of the systems of interest from single point calculations for sixteen different exchange-correlation functionals:

- an LDA functional, the Slater exchange in combination with local VWN correlation $(\mathrm{SVWN})[25]$

- the generalized gradient approximations (GGA) B97, [26] BLYP, [27, 28] BP86, [27, 29] OLYP, [28, 30] PBE [31] and PW91 [32]

- the hybrid B3LYP, [28, 33] B3P86, [29, 33] and B3PW91 [32, 33]

- the meta-GGA TPSS [34] and 
- the heavily parametrised Minnesota functionals M06, [35] M06-2X, [35] M06-L, [36] M06HF [37] and M11-L [38].

We also considered the Hartree-Fock, MP2 and CCSD wavefunction approximations. All the electronic structure calculations were performed with the GAMESS-US [39] and PySCF [40] packages. Later on, densities obtained from those single points were analysed and their corresponding eletronic energies partitioned under the IQA formalism, using our PROMOLDEN code. [41] The calculation used $\beta$-spheres with radii between 0.1 and 0.3a.u. Restricted angular Lebedev quadratures were used. Inside the $\beta$-spheres, we considered 451 mapped radial point trapezoidal quadratures and $L$ expansions truncated at $l=10$. Outside the $\beta$-spheres, we increased the number of mapped radial points to 651 and $L$ up to $l=12$. Finally, we visualised our results with the Python library Matplotlib [42] and the Avogadro program [43].

\section{Results}

In order to give a comprehensive perspective about the quality of the IQA descriptors using the exchange-correlation functionals addressed in this investigation, we assess the most relevant energetic features of the studied water clusters. Namely, we consider (i) cooperative effects in formation energies, (ii) IQA deformation and interaction energies and (iii) classical, exchangecorrelation and total interaction energies of covalent $\mathrm{O}-\mathrm{H}$ bonds. Later on, we examine the hydrogen bond hierarchy established by considering different structures of $\left(\mathrm{H}_{2} \mathrm{O}\right)_{6}$. We consider (iv) the classical and exchange-correlation energies and (v) QTAIM delocalisation indices of different types of hydrogen bond. The DFT functionals employed are tested against HF and MP2 references, which show similar behaviours. Due to the excessive computational resources requested to perform IQA analyses for medium and large-sized systems, comparisons with MP2 results without any approximation (like the one proposed by Müller [44]) cannot be extended to water hexamers. [20] Moreover, CCSD calculations were also performed to serve as a reference in the hydrogen bond cooperativity in homodromic structures.

\section{Hydrogen bond cooperative effects}

As discussed in the introduction, cooperativity is a prominent feature of hydrogen bonding homodromic water clusters, (i.e., $\mathrm{H}_{2} \mathrm{O}$ rings in which every single molecule forms two hydrogen bonds with two different molecules, being in one case the hydrogen donor and in the other, the hydrogen acceptor) are widely regarded as prototypical systems in the study of hydrogen 
bond cooperativity. [19] These effects are manifested in the mutual strengthening of hydrogen bonding within these structures.

A way to assess the hydrogen bond cooperativity in cluster formation energies is to compute the difference between the energy of the process $\left(\mathrm{H}_{2} \mathrm{O}\right)_{\mathrm{n}-1}+\mathrm{H}_{2} \mathrm{O} \rightleftarrows\left(\mathrm{H}_{2} \mathrm{O}\right)_{\mathrm{n}}\left(\Delta E_{\mathrm{n}}\right)$ and that of the water dimer, $\left(\Delta E_{2}\right)$ for which there is only one hydrogen bond,

$$
\Delta \Delta E=\Delta E_{n}-\Delta E_{2}
$$

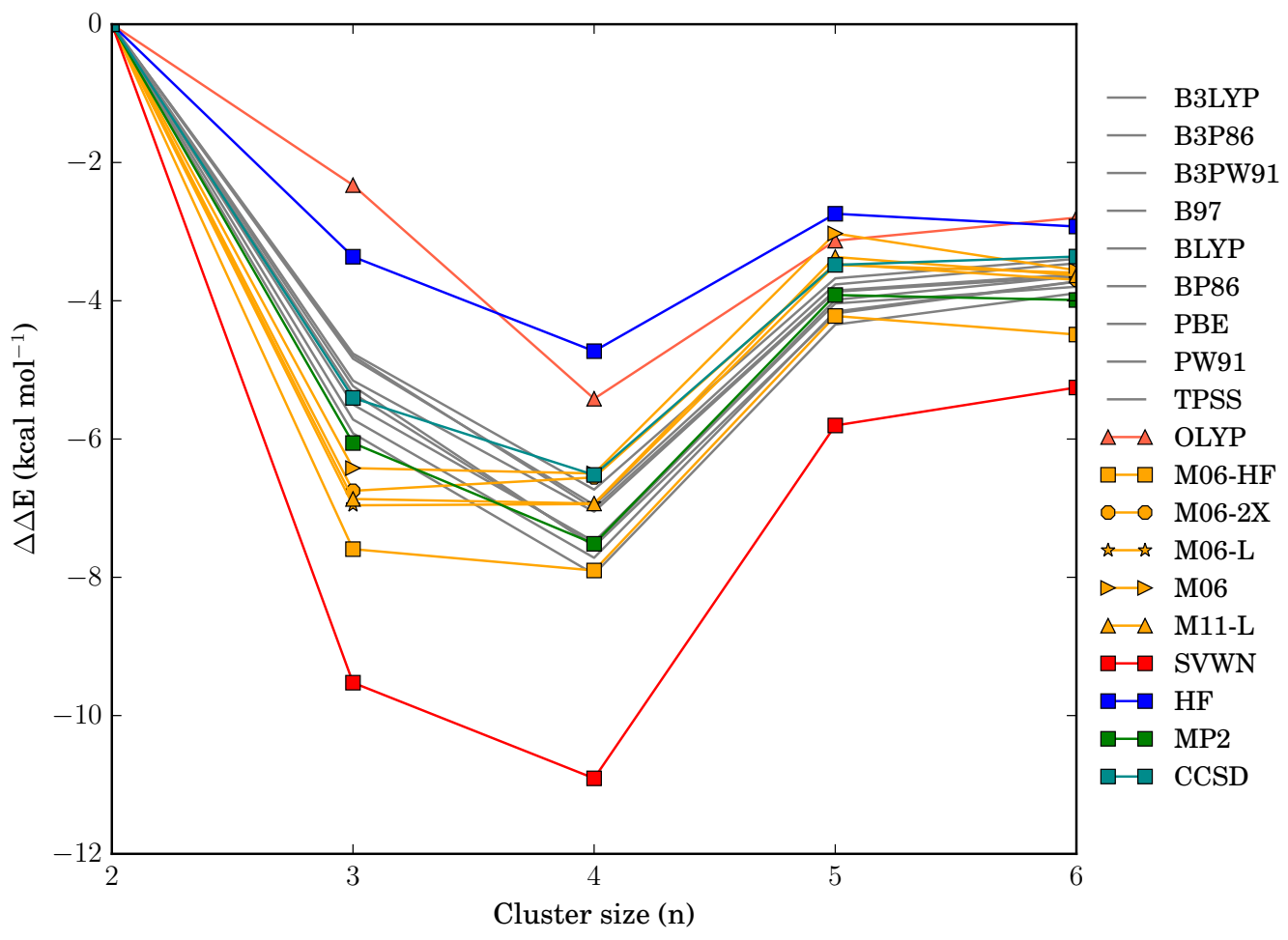

Figure 2: $\Delta \Delta E$ computed using Equation (18) with all the exchange-correlation functionals and wavefunction approximations considered in this investigation. Hydrogen bond cooperativity is indicated by negative values of $\Delta \Delta E$.

Figure 2 shows the computed values of $\Delta \Delta E$ as a function of the cluster size $(\mathrm{n}=2-6)$. We note that $\Delta \Delta E$ is negative in all cases, which means that all the considered approximations exhibit cooperativity in the examined homodromic water clusters. Concerning the wavefunction reference methods, HF, MP2 and CCSD, they all behave in a similar manner, resulting in trends and differences among them of a few $\mathrm{kcal} / \mathrm{mol}$. By considering CCSD calculations as reference, we can say that MP2 overestimates hydrogen bond cooperativity but to a lesser extent than HF 
underestimates it.

The CCSD reference behaviour is reproduced remarkably well by most DFT functionals tested. There are, however, some notable exceptions. First, the LDA-like functional SVWN strongly overestimates hydrogen bond cooperativity. This large deviation is not surprising given the simplicity of the LDA-like functional. Second, every Minnesota functional predict very similar values of hydrogen bond cooperativity for the water trimer and tetramer $\left(\Delta \Delta E_{3} \approx \Delta \Delta E_{4}\right)$, while for the CCSD reference, we observe that $\left|\Delta \Delta E_{4}\right|>\left|\Delta \Delta E_{3}\right|$. The reason behind this different behaviour may lie in the heavy parameterisation of the Minnesota family of functionals, although this should be further investigated. Finally, OLYP underestimates systematically the amount of cooperativity with respect to the CCSD results, in a very similar fashion to HF. This circumstance may result from the use of a different optimised exchange functional [30] in place of the standard one developed by Becke. [27]

\section{$I Q A$ 's deformation and interaction energies}

We can understand the formation of a molecular cluster as a three-step process within the IQA formalism. In the first step, the interacting monomers rearrange their nuclear positions to those in the complex. This usually leads to a small energy penalty known as preparation or strain energy. The electronic structure of the prepared monomers may then be envisaged to suffer a distortion (that includes polarization, charge transfer, electron delocalisation, etc) that leads to the final distribution as found in the complex. The sum of the energy penalties of these two first steps will be called here deformation energy $\left(E_{\text {def }}\right)$. [15] In the third step, the rearranged molecules interact to form the molecular cluster in what is denominated as interaction energy $\left(E_{\text {int }}\right) .[15]$ The value of $E_{\text {int }}$ for stable compounds is always negative. The formation energy of the molecular cluster is the sum of the deformation and interaction energies $\left(E_{\text {form }}=E_{\text {def }}+E_{\text {int }}\right)$. Figure 3 shows the deformation, interaction and formation energies per water molecule for the studied clusters. The trends are identical for all the considered approximations: $E_{\text {def }}$ and $\left|E_{\text {int }}\right|$ increase with the size of the cluster. Additionally, the magnitude of $E_{\text {int }}$ rises faster than $\left|E_{\text {def }}\right|$ with respecto to the number of interacting monomers (n), which results in an enlargement of $E_{\text {form }}$ with the size of the cluster. In all cases, the three quantities exhibit an asymptotic behaviour with $\mathrm{n}$.

The only noticeable deviation from the CCSD reference values of CCSD concerning hydrogen bond cooperative effects in small water clusters is the clear increase of $\left|E_{\text {form }}\right|$ by the functional 


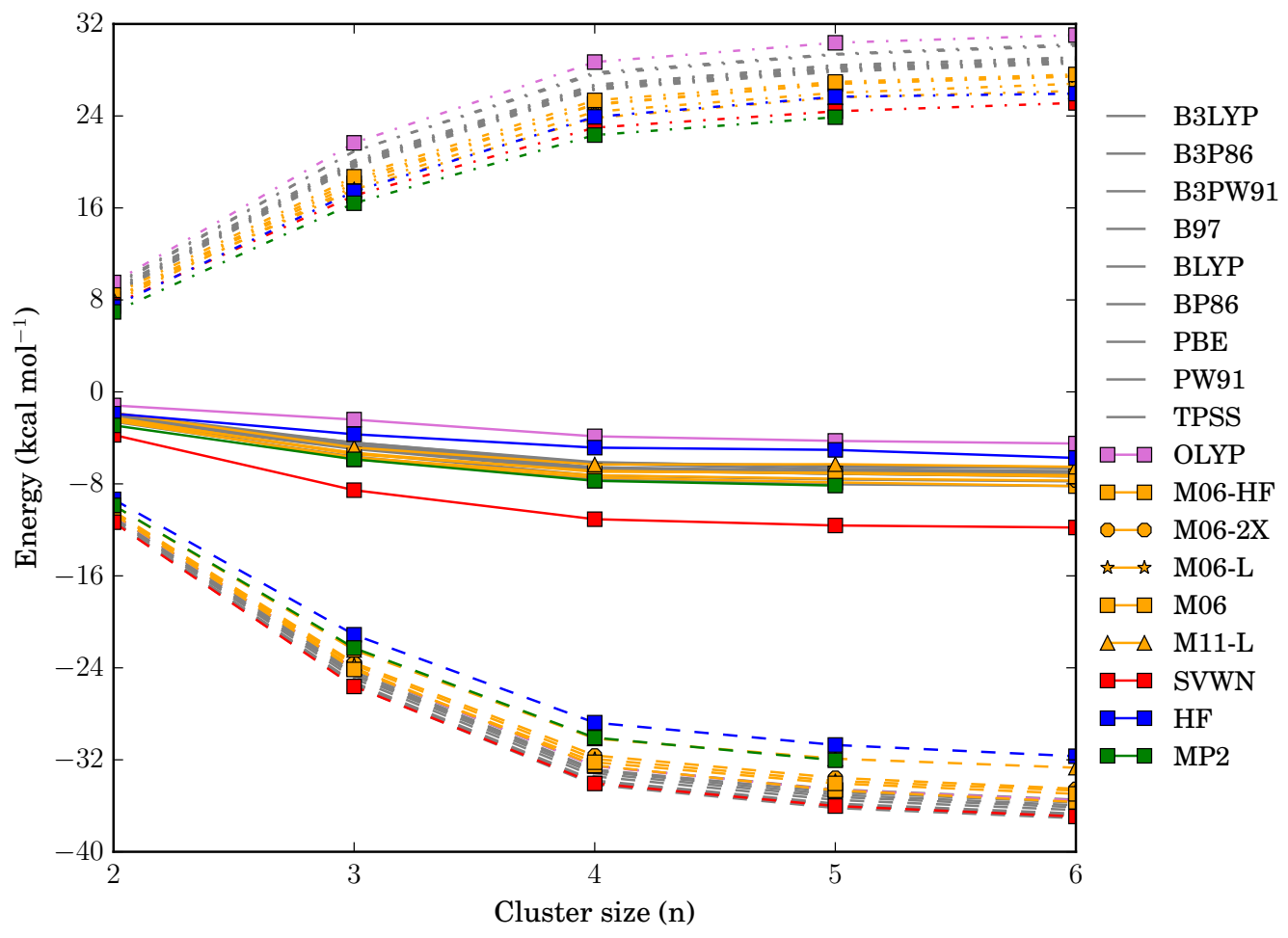

Figure 3: Formation (solid), deformation (dotted) and interaction energies (dashed lines) per water molecule in homodromic water clusters $\left(\mathrm{H}_{2} \mathrm{O}\right)_{\mathrm{n}}(\mathrm{n}=2-6)$.

SVWN. This circumstance results from a combination of an underestimation of the deformation energy, $E_{\text {def }}$, along with an overestimation of the interaction energy, $E_{\text {int }}$. The low values of $E_{\text {def }}$ are probably caused by the failure of SVWN to take into account the subtle changes ocurring in the deformation processes of the formation of the molecular clusters under consideration.

\section{Interaction energy of the $\mathrm{O}-\mathrm{H}$ covalent bond}

The effect of including extra water molecules to homodromic water clusters can be observed not only in the IQA interaction and deformation energies, but also in the intramolecular bond energies. In particular, the covalent $\mathrm{O}-\mathrm{H}$ bonds in which the hydrogen atom is $\mathrm{H}$-bonded to another water molecule are expected to suffer a significant change due to the aforementioned hydrogen bond cooperative effects. Figure 4 shows the differences in the interaction energy, and its exchange-correlation and classical components, for the $\mathrm{O}-\mathrm{H}$ covalent bond for the homodromic water clusters $\left(\mathrm{H}_{2} \mathrm{O}\right)_{n}$ with $\mathrm{n}=2-6$ with respect to the isolated monomer. The covalency of the $\mathrm{O}-\mathrm{H}$ interaction diminishes with the inclusion of an additional water monomer (dotted lines) with a slight underestimation of $\Delta V_{\mathrm{xc}}$ by the functional SVWN. We did not find 
any anomaly among the functionals beyond a moderate underestimation of $V_{\mathrm{xc}}$ by the LDA-like functional SVWN.

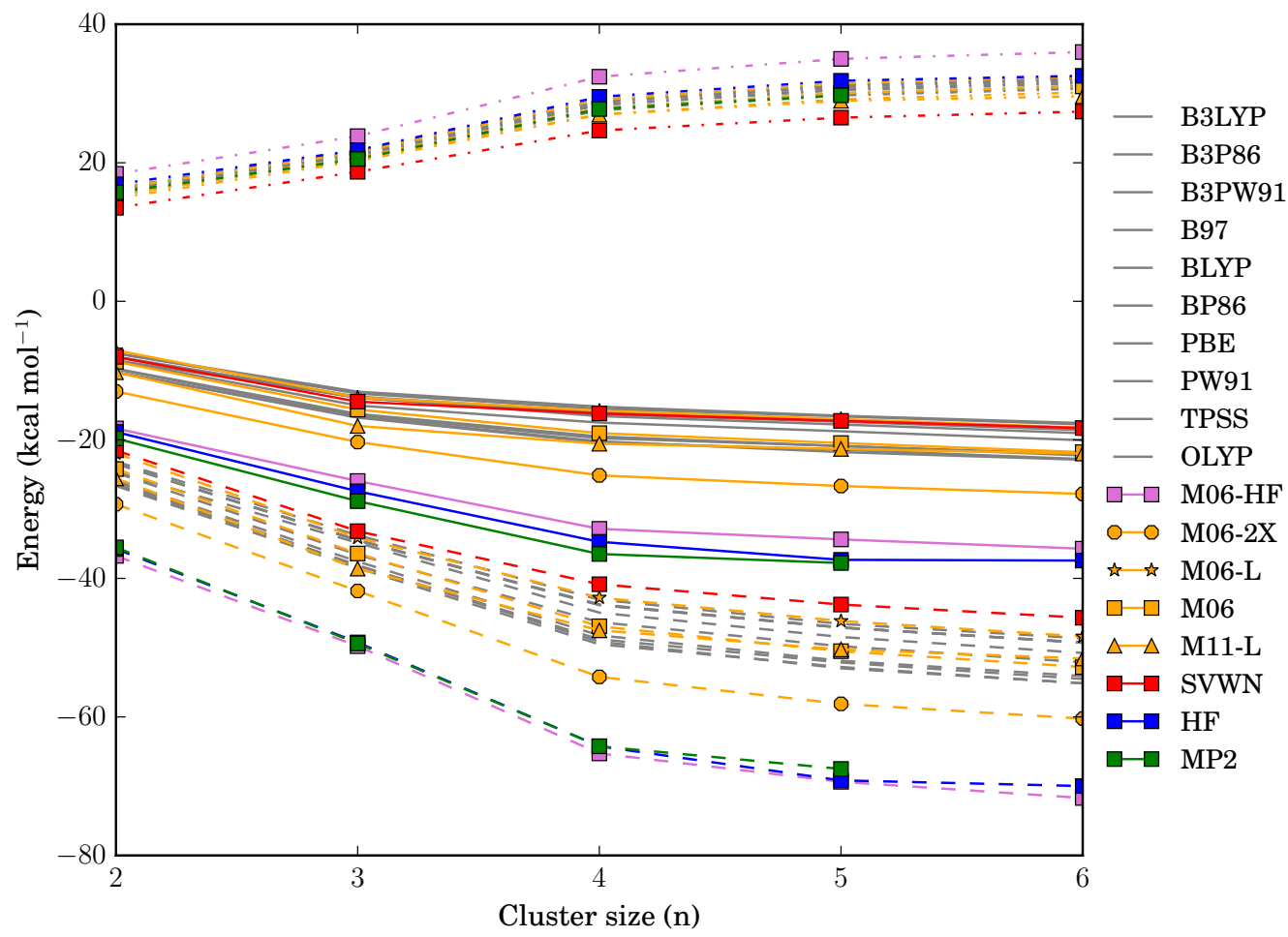

Figure 4: Differences in exchange-correlation (dotted), classical (dashed) and total interaction energies (solid) for $\mathrm{O}-\mathrm{H}$ bonds in which the hydrogen atom is $\mathrm{H}$-bonded to another water molecule within the homodromic clusters $\left(\mathrm{H}_{2} \mathrm{O}\right)_{\mathrm{n}}(\mathrm{n}=2-6)$. The values are given with respect to the monomer average energies.

Regarding the classical component of the interaction energy shown in Figure 4 with dashed lines, the magnitude of the charge of the $\mathrm{O}$ and hydrogen-bonded $\mathrm{H}$ basins increases when more molecules are included in the system. [19] Accordingly, the addition of water molecules makes the classical component of the $\mathrm{O}-\mathrm{H}$ interaction energy more stabilising, because of the larger charge of the involved atoms. Although all the approximations follow this trend, the MP2, HF, and the HF-like M06-HF results are clearly separated from the rest. For example, HF overestimates $\Delta V_{\mathrm{cl}}$. This overestimation might occur due to the overdelocalised electron density of the uncorrelated HF method, that leads to larger atomic charges than those computed in correlated calculations, and hence to an enlargement of the classical part of the interaction energy. [45] Finally, the magnitude of the overall change in the $\mathrm{O}-\mathrm{H}$ interaction energy in $\left(\mathrm{H}_{2} \mathrm{O}\right)_{n}$ clusters raises with $\mathrm{n}$, because $\left|\Delta V_{\mathrm{cl}}^{\mathrm{O}-\mathrm{H}}\right|>\left|\Delta V_{\mathrm{xc}}^{\mathrm{O}-\mathrm{H}}\right|$. Once again, the MP2, HF and M06-HF values are separated 
Table 1: Scale of hydrogen bond formation energies within water clusters proposed in this study. The hierarchy is presented in an increasing order of magnitude. Taken from reference [20].

\begin{tabular}{|c|c|}
\hline Type of HB & Description \\
\hline (1) & $\begin{array}{l}(i) \text { the } \mathrm{H} \text { atom involved in the hydrogen bond belongs to a double } \mathrm{HB} \\
\text { donor and }(i i) \text { the oxygen that participates in the interaction acts as a } \\
\text { double } \mathrm{HB} \text { acceptor. }\end{array}$ \\
\hline$(2)$ & $\begin{array}{l}(i) \text { the hydrogen of a double } \mathrm{HB} \text { donor is bonded to the oxygen of a } \\
\text { single } \mathrm{HB} \text { acceptor or }(i i) \text { the oxygen of a double acceptor interacts with } \\
\text { a hydrogen of a single donor. }\end{array}$ \\
\hline$(3)$ & $\begin{array}{l}\text { a hydrogen bond is formed between two double HB donors or two double } \\
\text { HB acceptors. }\end{array}$ \\
\hline (4) & $\begin{array}{l}\text { a hydrogen of a single } \mathrm{HB} \text { donor is bonded to the oxygen of a single } \mathrm{HB} \\
\text { acceptor. }\end{array}$ \\
\hline (5) & $\begin{array}{l}(i) \text { a hydrogen of a double } \mathrm{HB} \text { acceptor is in contact with the oxygen } \\
\text { of a single donor or }(i i) \text { the } \mathrm{O} \text { atom of a double donor interacts with a } \\
\text { hydrogen of a single acceptor. }\end{array}$ \\
\hline (6) & $\begin{array}{l}\text { the oxygen of a double HB donor interacts with a hydrogen of a double } \\
\text { HB acceptor }\end{array}$ \\
\hline
\end{tabular}

from the rest of approximations considered herein, which describe $\Delta E_{\text {int }}^{\mathrm{O}-\mathrm{H}}$ in a very similar way.

\section{Types of hydrogen bonds}

The water clusters studied so far are prototypical systems where only hydrogen-bond cooperativity takes place. We can also consider anticooperative effects (i.e., the weakening that the ocurrence of certain hydrogen bonds cause on others) which appear in water hexamers different from the ring structure. We suggested previously a hydrogen bond strength hierarchy based on the coordination number of the hydrogen acceptor and donor involved in the interaction. [20] This hierarchy is shown in Table 1 . We assess now this hierarchy with the different functionals considered herein.

Figure 5 depicts the classical and exchange-correlation parts of $E_{\text {int }}^{\prime}$ from Equation (7). In all cases, the dominant term is the exchange-correlation contribution (in solid lines). The classical part of $E_{\text {int }}^{\prime}$ is much smaller in absolute value but still an attractive contribution for all the 


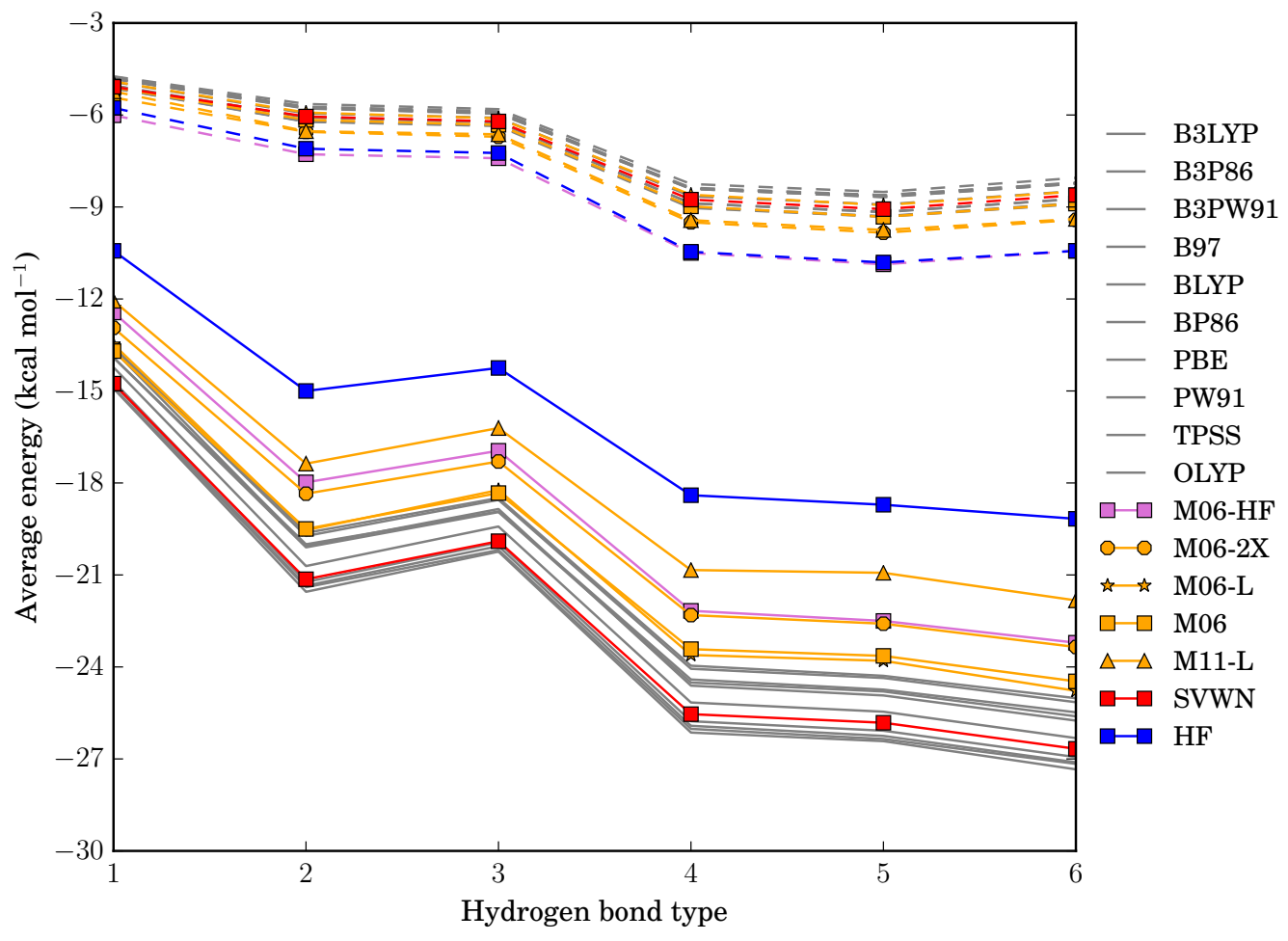

Figure 5: Average values of the classical (dashed) and exchange-correlation (solid lines) contributions to the interaction energy between water molecules computed with the aid of Equation (7).

examined exchange-correlation functionals. The trends in classical and exchange-correlation components match overall those reported in reference [20]. The only appreciable disparity concerns the exchange-correlation energies of the hydrogen bonds of types 2 and 3 (Table 1) whose hydrogen bond strength are in the inverse order with respect to those previously reported in reference [20]. Another noticeable feature of Figure 5 is the appreciable underestimation of the exchange-correlation energy by the HF method. To further assess these results, we computed the delocalisation indexes (DI) between the water molecules within the addressed water hexamers. Figure 6 shows the average value of the delocalisation indices for the different types of hydrogen bond for each functional and for HF. We observe again that hydrogen bonds of type 2 had a larger covalent component. This in contrast with out previous results. [20]

Despite the slight discrepancies observed in the description of hydrogen cooperative and anticooperative effects, most of the exchange-correlation functionals considered herein describe suitably the hydrogen bond non-additivity within water clusters. This results paves the wave for the utilisation of the addressed functionals in the study of larger warter clusters and hydrogen- 


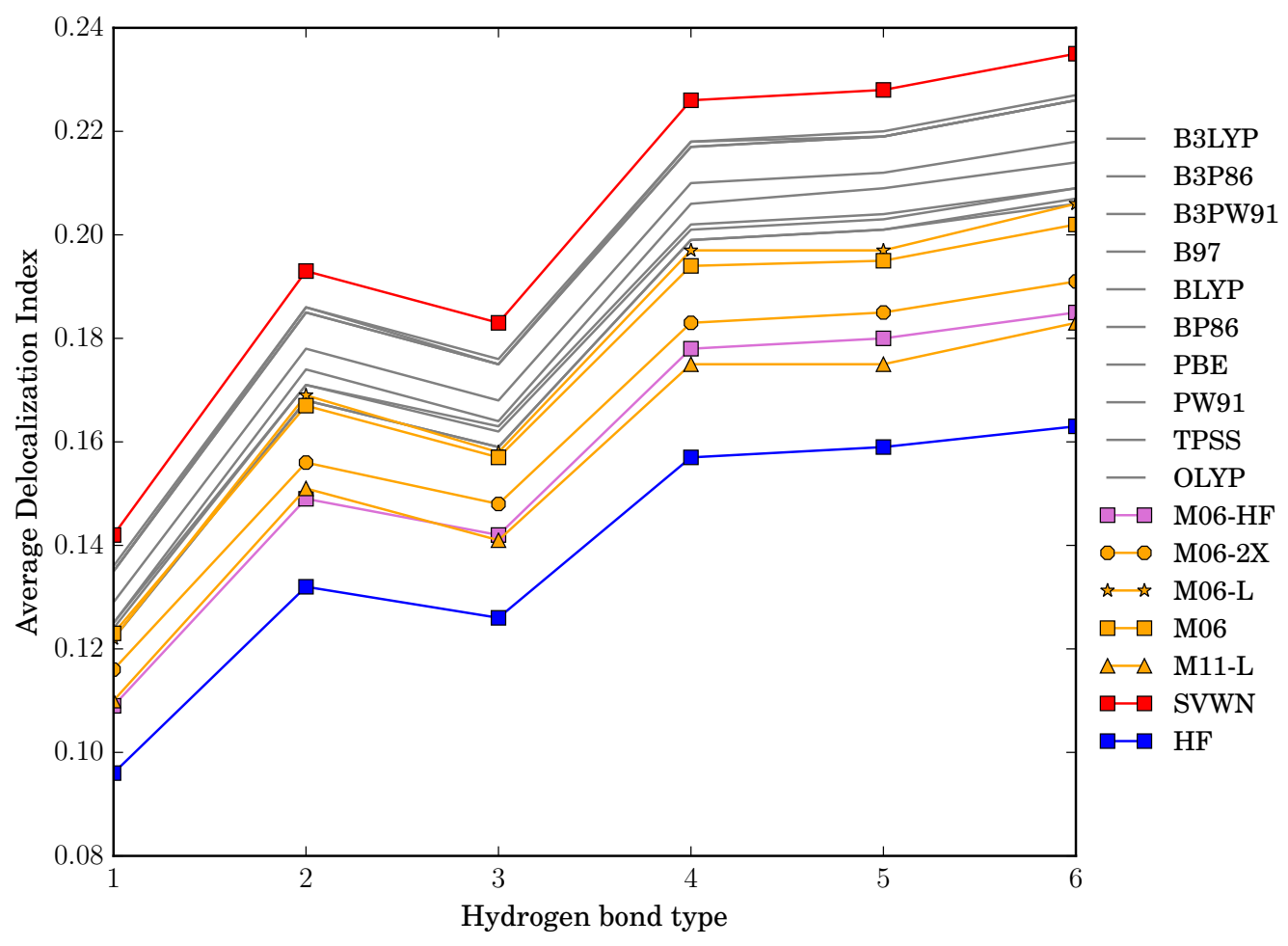

Figure 6: Averages of the delocalisation indices for the type of hydrogen bonds in Table 1.

bonded molecular clusters in general.

\section{Conclusions}

We have assessed the ability of a variety of DFT functionals to describe hydrogen bond cooperative and anticooperative effects in different water clusters. Our results indicate that most functionals correctly characterise the tendencies of the IQA partition of the electronic energy computed with ab-initio methodologies. All but the SVWN, OLYP and Minnesota's functionals reproduce the hydrogen bond cooperativity profile of the reference. Only the SVWN overestimates the interaction energy in an appreciable manner, and the interactions $\mathrm{O}-\mathrm{H}$ and $\mathrm{O} \cdots \mathrm{H}$ are adequately reproduced, being the HF-like M06-HF the closest functional to the HF and MP2 references. When applied to the hydrogen bond hierarchy classification, the same conclusions are found. Regarding the decrease in the quality of the electron densities reported by Medvedev et al., [22] we have not found any appreciable effect in the newest functionals, at least as the description of the non-additive effects in the hydrogen bonds addressed in this investigation is regarded. Overall, we hope that this study represents a basis for the application 
of IQA/DFT to a larger vaierty of chemical systems, such as, large water clusters.

\section{Acknowledgements}

We thank the spanish MINECO, grant CTQ2015-65790-P, the FICyt, grant IDI-2018-000177 and the European Union FEDER funds for financial support. F. J.-G. gratefully acknowledge financial support from the Spanish MINECO, grant BES-2016-076986. T.R.R. acknowledges financial support from CONACyT/Mexico (grant 253776).

\section{References}

[1] A. D. Becke, J. Chem. Phys. 2014, 140.

[2] H. S. Yu, S. L. Li, D. G. Truhlar, J. Chem. Phys. 2016, 145.

[3] R. Galindo-Murillo, M. E. Sandoval-Salinas, J. Barroso-Flores, J. Chem. Theory Comput. 2014, 10, 825834.

[4] A. J. Cohen, P. Mori-Sánchez, W. Yang, Chem. Rev. 2012, 112, 289-320.

[5] D. Lupp, N. J. Christensen, J. R. Dethlefsen, P. Fristrup, Chem. Eur. J. 2015, 21, 3435-3442.

[6] P. Geerlings, F. De Proft, Phys. Chem. Chem. Phys. 2008, 10, 3028-3042.

[7] R. A. Miranda-Quintana, P. W. Ayers, Theor. Chem. Acc. 2019, 138, 44.

[8] M. Franco-Pérez, C. A. Polanco-Ramírez, J. L. Gázquez, P. W. Ayers, J. Mol. Model. 2018, 24, 285.

[9] F. Cortés-Guzmán, R. Bader, Coord. Chem. Rev. 2005, 249, 633-662.

[10] E. Romero-Montalvo, J. M. Guevara-Vela, W. E. Vallejo Narváez, A. Costales, A. Martín Pendás, M. Hernández-Rodríguez, T. Rocha-Rinza, Chem. Commun. 2017, 53, 3516-3519.

[11] R. F. W. Bader, P. M. Beddall, J. Chem. Phys. 1972, 56, 3320-3329.

[12] A. D. Becke, K. E. Edgecombe, J. Chem. Phys. 1990, 92, 5397-5403.

[13] C. Gatti, F. Cargnoni, L. Bertini, J. Comput. Chem. 2003, 24, 422-436.

[14] P. L. A. Popelier, Applications of Topological Methods in Molecular Chemistry, Springer International Publishing, 2016.

[15] M. Á. Blanco, Á. Martín Pendás, E. Francisco, J. Chem. Theory Comput. 2005, 1, 1096-1109.

[16] E. Francisco, Á. Martín Pendás, M. Á. Blanco, J. Chem. Theory Comput. 2006, 2, 90-102. 
[17] P. Maxwell, Á. Martín Pendás, P. L. A. Popelier, Phys. Chem. Chem. Phys. 2016, 18, 20986-21000.

[18] E. Francisco, J. L. Casals-Sainz, T. Rocha-Rinza, Á. Martín Pendás, Theor. Chem. Acc. 2016, 135.

[19] J. Guevara-Vela, R. Chávez-Calvillo, M. García-Revilla, J. Hernández-Trujillo, O. Christiansen, E. Francisco, Á. MartínPendás, T. Rocha-Rinza, Chem. Eur. J. 2013, 19, 14304-14315.

[20] J. Guevara-Vela, E. Romero-Montalvo, V. Mora Gómez, R. Chávez-Calvillo, M. García-Revilla, E. Francisco, Á. Martín Pendás, T. Rocha-Rinza, Phys. Chem. Chem. Phys. 2016, 18, 19557-19566.

[21] M. J. Gillan, D. Alfè, A. Michaelides, J. Chem. Phys. 2016, 144, 130901.

[22] M. G. Medvedev, I. S. Bushmarinov, J. Sun, J. P. Perdew, K. A. Lyssenko, Science 2017, 355, 49-52.

[23] R. Bader, Atoms in molecules : a quantum theory, Clarendon Press, Oxford New York, 1990.

[24] J. Segarra-Martí, M. Merchan, D. Roca-Sanjuan, J. Chem. Phys. 2012, 136, 244306.

[25] S. H. Vosko, L. Wilk, M. Nusair, Can. J. Phys. 1980, 58, 1200-1211.

[26] A. D. Becke, J. Chem. Phys. 1997, 107, 8554-8560.

[27] A. D. Becke, J. Chem. Phys. 1988, 88, 2547.

[28] C. Lee, W. Yang, R. G. Parr, Phys. Rev. B 1988, 37, 785-789.

[29] J. P. Perdew, Phys. Rev. B 1986, 33, 8822-8824.

[30] N. A. Handy, A. J. Cohen, Mol. Phys. 2001, 99, 403-412.

[31] J. P. Perdew, K. Burke, M. Ernzerhof, Phys. Rev. Lett. 1996, 7r, 3865-3868.

[32] J. P. Perdew, J. A. Chevary, S. H. Vosko, K. A. Jackson, M. R. Pederson, D. J. Singh, C. Fiolhais, Phys. Rev. B 1992, 46, 6671-6687.

[33] A. D. Becke, J. Chem. Phys. 1993, 98, 5648.

[34] J. Tao, J. P. Perdew, V. N. Staroverov, G. E. Scuseria, Phys. Rev. Lett. 2003, 91, 146401.

[35] Y. Zhao, D. G. Truhlar, Theor. Chem. Acc. 2008, 120, 215-241.

[36] Y. Zhao, D. G. Truhlar, J. Chem. Phys. 2006, 125, 194101.

[37] Y. Zhao, D. G. Truhlar, J. Phys. Chem. A 2006, 110, 13126-13130.

[38] R. Peverati, D. G. Truhlar, J. Phys. Chem. Lett. 2012, 3, 117-124.

[39] M.W.Schmidt, K.K.Baldridge, J.A.Boatz, S.T.Elbert, M.S.Gordon, J.J.Jensen, S.Koseki, N.Matsunaga, K.A.Nguyen, S.Su, T.L.Windus, M.Dupuis, J.A.Montgomery, J. Comput. Chem. 1993, 14, 1347. 
[40] Q. Sun, T. C. Berkelbach, N. S. Blunt, G. H. Booth, S. Guo, Z. Li, J. Liu, J. D. McClain, E. R. Sayfutyarova, S. Sharma, S. Wouters, G. K.-L. Chan, Wiley Interdiscip. Rev. Comput. Mol. Sci. 2018, 8, e1340.

[41] Á. Martín Pendás, E. Francisco, Promolden. A QTAIM/IQA code (unpublished).

[42] J. D. Hunter, Computing In Science \& Engineering 2007, 9, 90-95.

[43] M. D. Hanwell, D. E. Curtis, D. C. Lonie, T. Vandermeersch, E. Zurek, G. R. Hutchison, J. Cheminformatics 2012, $4,17$.

[44] A. Müller, Phys. Lett. A 1984, 105, 446-452.

[45] J. Thirman, M. Head-Gordon, J. Phys. Chem. Lett. 2014, 5, 1380-1385. 